\title{
Dynamic Modeling of a Boring Bar Using Theoretical and Experimental Engineering Methods Part 2: Finite Element Modeling and Sensitivity Analysis
}

\author{
Tatiana Smirnova \\ Department of Signal Processing, Blekinge Institute of Technology, PO Box 520, SE-37225 Ronneby, Sweden
}

\author{
Henrik Åkesson \\ Department of Signal Processing, Blekinge Institute of Technology, PO Box 520, SE-37225 Ronneby, Sweden \\ Acticut International AB, Gjuterivägen 7, 31132 Falkenberg, Sweden
}

\section{Lars Håkansson}

Department of Signal Processing, Blekinge Institute of Technology, PO Box 520, SE-37225 Ronneby, Sweden

\begin{abstract}
This is the second of two companion papers that summarize the theoretical and experimental work carried out concerning modeling of dynamic properties of boring bars. This paper introduces the finite element method for the modeling of clamped boring bars. The " 3 -D" FE models of the system boring bar - clamping house as well as the "1-D" FE models of the clamped boring bar were derived. In particular, the modeling of the boring bar clamping is addressed. Dynamic properties predicted based on the developed FE models of the clamped boring bar were compared with the ones estimated by means of experimental modal analysis conducted on the actual boring bar clamped in the lathe. The "3-D" FE models display substantially higher correlation with the experimental modal analysis results compared to the "1-D" FE models. A "3-D" FE model of the boring bar - clamping house manages to model the distance in frequency and the orientation of the two fundamental modes to a large extent. The importance of the modeling of the boring bar boundary conditions for the accuracy of dynamic models of boring bars is demonstrated. The sensitivity of the natural frequency estimates produced by means of the FE and the continuous system (presented in Part 1) boring bar models with respect to variations in material density and Young's elastic modulus has been addressed.
\end{abstract}

\section{INTRODUCTION}

This paper is focused on modeling of dynamic properties of clamped boring bars by means of finite element method. A number of research works have been completed that includes the modeling of a boring bar as a system with a large but finite number of degrees of freedom, using finite element analysis. Wong et al. used Timoshenko beam finite elements to model a boring bar. ${ }^{1} \mathrm{He}$ designed an electromagnetic dynamic absorber and simulated its performance, utilizing modal control and direct feedback control based on the finite element model of the boring bar. Wong claims that both controllers succeed in significant damping of boring bar vibrations at the lowest vibration mode. Nagano et al. used pitched-based carbon-fiber reinforced plastic (CFRP) material to develop a chatter-resistant boring bar with a large overhang. ${ }^{2}$ He made an attempt to create a "3-D" finite element model in order to predict natural frequencies and improve dynamic characteristics of the boring bar by modeling embedded steel cores of various shapes. The cutting performance and stability of the designed boring bars regarding chatter were investigated experimentally. He claims that utilization of the CFRP material with the crossshaped steel core allows for the successful, stable machining for boring bars with a length-to-diameter of more than seven.
Nagano also mentioned the necessity concerning the development of improved models for the clamping of the boring bar. Baker et al. used FEM in the stability analysis of a turning operation. ${ }^{3} \mathrm{He}$ approximated the cutting force by using the orthogonal cutting model. Baker also based his method of stability prediction on the assumption of the linearity of the tooling structure's behavior. Two different types of FE models were considered: first, "1-D” FE models of a boring bar and a workpiece attached to a rigid base, and second, "3-D" FE models of a boring bar and a workpiece attached to a deformable "3-D" FE model of machine tool. The FE models were used to extract structural matrices, with the purpose of using them in stability analysis. The maximum stable width of cut was predicted for a set of geometric dimensions of the tool holder and the workpiece (for both types of models) as a function of spindle speed. However, no experimental results were presented.

In addition, a number of research works have been completed concerning external longitudinal turning in which the tool holder shank, etc. have been modeled using the finite element method. Sturesson et al. developed a "3-D" finite element model of a tool holder shank - clamping house for external longitudinal turning. ${ }^{4}$ They used normal mode analysis to evaluate the natural frequencies, modal masses, and mode participation factors of the tool holder shank. The modal damping 